\title{
¿Por qué es necesario repensar la competencia docente para el mundo digital?
}

\section{Why rethinking teaching competence for the digital world?}

\author{
Linda Castañeda \\ Universidad de Murcia, Murcia. España \\ lindacq@um.es \\ Francesc Esteve \\ Univeristat Jaume I, Castellón de la Plana. España \\ festeve@uji.es \\ Jordi Adell \\ Univeristat Jaume I, Castellón de la Plana. España \\ jordi@uji.es
}

\begin{abstract}
Resumen
En este artículo se analizan los modelos institucionales de competencia digital docente con mayor impacto en los últimos tiempos y se abordan críticamente tres características comunes a todos ellos, entendidas como deficiencias en su planteamiento. En primer lugar, que no parten explícitamente de un modelo de acción docente y de manera implícita reducen la función docente al trabajo en el aula, evitando aspectos como el compromiso social y político o el papel de la escuela en el desarrollo comunitario. En segundo lugar, que parten de una visión taxonómica del concepto de competencia, ignorando la complejidad de los diferentes contextos en los que dicha competencia se pone en acción y se desarrolla y en su papel en la construcción de la identidad del docente, algo que enfoques sociomateriales y holísticos sí incluyen. En tercer lugar, que se fundamentan de manera contradictoria en una visión instrumentalista de la tecnología como herramienta neutra en valores y, por el contrario, en una concepción determinista de la relación entre tecnología y sociedad. Finalmente, se propone a debate un modelo que hemos denominado "Competencia Docente Integral en el mundo digital”, específicamente diseñado para la educación básica, que está siendo objeto de una investigación más ambiciosa

Palabras clave

Competencia docente, competencia digital docente, acción docente, competencia holística, desarrollo profesional docente.
\end{abstract}

\footnotetext{
Abstract

This article analyses the most impacting institutional models of digital teaching competence, and critically addresses three characteristics common to all of them, understood as deficiencies in their approach. Firstly, they do not explicitly start from a model of teaching action, and implicitly reduce the teaching function to the classroom performance, avoiding aspects such as social and political commitment, or the role of the school in community development. Secondly, they are based on a taxonomic vision of the concept of competence, ignoring the complexity of the different contexts in which competence is carried on and developed, as well as its role in the construction of teacher identity, something that socio-material and holistic approaches do include. Thirdly, they are based on an instrumentalist vision of technology as a neutral tool in values and, on the contrary, on a deterministic conception of the relationship between technology and society. Finally, we present a model that we have called "Integral Teaching Competence for the digital world", specifically designed for basic education, which is the subject of a more ambitious research.

Key words

Teaching Competence, Digital teaching competence, Teaching action, Teaching Professional Development.
} 


\section{Introducción}

Nuestra sociedad ha experimentado en las últimas décadas profundas transformaciones sociales, económicas y culturales. Preparar a los jóvenes para vivir en el complejo mundo actual y para afrontar con garantías un futuro incierto son algunos de los mayores desafíos de la educación actual. Las nuevas tecnologías de la información y la comunicación (TIC) han sido uno de los factores de mayor impacto en todos los sectores de la actividad humana. Han revolucionado la producción y distribución de bienes y servicios, las relaciones humanas y la propia cultura. Pero su potencial en educación todavía está por desarrollar y demostrar.

"Las tecnologías de la información y la comunicación (TIC) pueden contribuir al acceso universal a la educación, a la equidad en la educación, a la enseñanza y el aprendizaje de calidad, al desarrollo profesional de los docentes y a una gestión, gobernanza y administración educativas más eficientes" (UNESCO, 2014; énfasis añadido).

Uno de los factores clave de su integración -no entramos ahora en el análisis del trasfondo de ésta visón del papel de las TIC en educación- es la formación del profesorado, por ejemplo, sus conocimientos sobre contenidos, pedagogía y tecnología (Mishra, Koehler y Henriksen, 2011). Numerosos autores han insistido en la necesidad de formar mejor a los docentes en el conjunto de conocimientos, habilidades y actitudes necesarios para promover el aprendizaje del alumno en un mundo crecientemente tecnológico (Carrera y Coiduras, 2012; Hall, Atkins y Fraser, 2014; Krumsvik, 2009 y 2012). Es decir, se enfatiza la necesidad de adaptación del contexto educativo a las necesidades que, supuestamente, nos plantean las tecnologías. Bien entrado el siglo XXI, la formación docente en tecnologías de la información y la comunicación supone todavía un reto tanto en la formación inicial, como en el desarrollo profesional de los docentes. Para hacer frente a ese reto ha surgido en los últimos años el concepto de competencia digital docente (en adelante CDD).

Existen diferentes marcos conceptuales y modelos a nivel internacional que han tratado de definir la CDD (Esteve, 2015). En los últimos años se han publicado diferentes estudios que analizan y comparan dichos marcos, tanto los referidos al profesorado de educación básica (Álvarez y Gisbert, 2015; Esteve y Gisbert, 2013; Pettersson, 2017), como del profesorado universitario ((Durán, Gutiérrez, y Prendes, 2016; Prendes y Gutiérrez, 2013).

Más allá de las diferencias aparentes entre los distintos modelos, derivadas principalmente del contexto, del nivel educativo o del propósito con el que fueron diseñados, en general todos ellos parten de la tecnología y de los cambios que esta supone para acción humana en general o para los modelos productivos en particular y, sobre ello, colocan una "capa" pedagógica que "redirige" la competencia hacia el contexto educativo. Esa "capa", que en nuestra opinión suele ser bastante fina, en el mejor de los casos se basa en una visión simplificada de la educación, reduciéndola a la actuación en el aula y en la que, en el mejor de los casos, el papel del docente suele restringirse al de enseñante de un alumno individual en colectividad. Ese tipo de aproximación a la CDD desde la tecnología como base fundamental y con esa fina capa pedagógica, ha traído consecuencias importantes e indeseables que se han convertido en invisibles para aquellos que usamos esos modelos: un modelo docente (la capa pedagógica) poco definido y lineal, una visión neutra e ingenua de la tecnología y una

¿Por qué es necesario repensar la competencia docente para el mundo digital? Linda Castañeda, Francesc Esteve y Jordi Adell.

Página 2 de 20 
definición de competencia basada en las necesidades instrumentales de los procesos productivos que rehúye las dimensiones más sociales de la definición de competencia.

La importancia de estos marcos deviene del hecho se utilizan como referentes y justificación de decisiones en la formación inicial y permanente del profesorado o en la certificación exigida para el ejercicio de la profesión docente, entre otros usos. Sus procesos de elaboración, apoyados generalmente en la consulta a docentes, eso sí, "guiada" por grupos de expertos, legitima su estatus profesional y esconde el hecho evidente de que, en gran medida, responden a preocupaciones e intereses corporativos. Un ejemplo de ello es la insistencia, durante años, en incluir el "uso legal de la Internet", es decir, en acabar desde la escuela con el fenómeno de la, así llamada, "piratería".

A continuación, proponemos un análisis exploratorio de algunos modelos de CDD. Como hemos dicho, somos conscientes de que no son pocas las aproximaciones que se han hecho de la competencia digital. Sin embargo, en este análisis y desarrollo que nos ocupa, y con un afán de dar el valor que merece al carácter situado de la competencia del que hablaremos más adelante, además de centrarnos en aquellos modelos institucionales de enseñanzas preuniversitarias, trataremos de abundar en propuestas a esos tres problemas detectados en ese análisis, o lo que es lo mismo, abundaremos en qué significa un concepto más holístico, integral y situado de competencia, una comprensión de la tecnología que supere la visión netamente instrumentalista imperante y una visión limitada de la docencia. Finalmente, y a modo de conclusión, propondremos de modo tentativo un modelo holístico de competencia docente para el mundo digital basado en una perspectiva más amplia y compleja de la acción docente, una perspectiva que creemos que responde mejor a la complejidad y amplitud presente y futura de la profesión docente.

\section{Los modelos institucionales de competencia digital docente}

\subsection{Metodología}

Se ha realizado un estudio descriptivo (Salkind, 2018) de los que se consideran los grandes marcos institucionales que definen la competencia digital del profesorado en la actualidad. Esta descripción da lugar a un análisis en el que se realiza una contextualización de cada uno de los marcos, se describen sus desarrollos derivados y se realiza una clasificación de sus componentes, que pretende fundamentar el análisis crítico y la discusión posteriores.

No todos los modelos de CDD son normativos o han sido publicados por autoridades educativas, pero algunos aspiran a ello en el momento presente y todos sirven de fundamento intelectual y justificación a desarrollos legislativos en el área de su influencia relacionados con la certificación de la competencia, la formación inicial y permanente del profesorado o la promoción del profesorado, entre otros temas. Por lo mismo, en este estudio se ha realizado un muestreo de conveniencia (Salkind, 2018), en el que se han seleccionado aquellos marcos de especial relevancia internacional (cinco) y nacional (dos) que pretenden, por la institución que los promulga, tener un mayor impacto tanto en diseño de la legislación, como en el desarrollo de la política educativa relacionada con ese particular.

¿Por qué es necesario repensar la competencia docente para el mundo digital? Linda Castañeda, Francesc Esteve y Jordi Adell.

Página 3 de 20 


\subsection{Los marcos institucionales estudiados}

A nivel internacional, aunque con base en Estados Unidos, uno de los modelos más conocidos e influyentes es el National Educational Technology Standards for Teachers (NETS-T), propuesto por la International Society for Technology in Education (ISTE, 2008). NETS-T incluye cinco dimensiones que desarrolla en una serie de indicadores de desempeño. NETS-T se centra en cómo los docentes pueden facilitar el aprendizaje de sus alumnos a través de las TIC, abordando desde la parte más didáctica del uso de la tecnología en el aula (diseño y evaluación de materiales y actividades), hasta aspectos de ciudadanía digital y desarrollo profesional docente. Este modelo ha sido recientemente actualizado (ISTE, 2017) y su desarrollo dista enormemente de la propuesta anterior, poniendo ahora el foco en la importancia de que los estudiantes piensen por sí mismos y conduzcan su propio aprendizaje. Para ello, establecen una serie de perfiles y habilidades para que el docente esté capacitado y sea el "catalizador" de ese aprendizaje.

Otro de los modelos más conocidos a nivel internacional es el propuesto por UNESCO (2008, 2011), que plantea un marco general para el establecimiento de estándares en competencias TIC para docentes, tanto en activo, como en formación. Un modelo con un enfoque netamente organizacional que, además de los aspectos didácticos, aborda cuestiones como la integración de las TIC en el plan de estudios, en la organización y administración o en la formación profesional de los docentes.

A nivel europeo, el Joint Research Centre (JRC) de la Comisión Europea (European Commission, 2017) se encuentra, en el momento de cerrar esta publicación, acabando de elaborar el Marco Europeo para la Competencia Digital del Profesorado $($ DigCompEdu). Se trata de un modelo que incluye aspectos relacionados con el compromiso profesional del profesorado y su entorno profesional, el uso de las TIC en el proceso de enseñanza-aprendizaje o el empoderamiento de sus estudiantes y el desarrollo de su propia competencia digital como ciudadanos.

Diferentes países han desarrollado también sus propios marcos y modelos. Por ejemplo, el modelo chileno propuesto por Enlaces (2011) establece cinco dimensiones que corresponden a funciones clave que desarrolla un docente en cuanto a la integración de la tecnología. Por su parte, y de una manera similar, el modelo británico conocido como DigiLit (Fraser, Atkins, y Hall, 2013) identifica seis áreas clave en la formación de los docentes incluyendo aspectos básicos de su trabajo en la era digital: búsqueda de información, comunicación, seguridad, creación y evaluación de recursos y actividades, entornos de aprendizaje y desarrollo profesional.

A nivel español hay que destacar, en primer lugar, el Marco Común de Competencia Digital Docente del Instituto Nacional de Tecnologías Educativas y de Formación del Profesorado (INTEF, 2013 y 2017), que toma como base el modelo de competencia digital genérica o ciudadana DigComp de la Comisión Europea (Ferrari, 2013) al que añade diversos aspectos pedagógicos. Algunas comunidades autónomas, como por ejemplo Andalucía, Extremadura o el País Vasco tomaron este modelo como referencia, proponiendo diferentes desarrollos derivados. Por otro lado, en 2016 el Departament d'Ensenyament de la Generalitat de Catalunya publicó su propio marco para la definición de la CDD que incluía habilidades tecnológicas de tipo instrumental y de tipo didáctico y metodológico (Departament d'Ensenyament, 2016).

¿Por qué es necesario repensar la competencia docente para el mundo digital? Linda Castañeda, Francesc Esteve y Jordi Adell.

Página 4 de 20 
Todos estos modelos han dado lugar a diferentes desarrollos e implementaciones a lo largo de los años. Den los EE.UU., por ejemplo, a partir del modelo NETS-T de ISTE (2008) un gran números de estados han elaborado sus propios estándares y se han publicado diferentes libros y manuales para facilitar su comprensión o guiar su implementación en las escuelas (Morphew, 2012). Algo que también ha sucedido con el modelo chileno Enlaces $(2011,2013)$. El modelo de ISTE ha servido también de referencia para múltiples investigaciones acerca del nivel de competencia digital del profesorado en activo o en formación (Banister y Reinhart, 2012; Esteve, Cela, y Gisbert, 2016; Kumar y Vigil, 2011). Modelos como el de UNESCO (2011) han sido tomados como referencia para el diseño de planes estratégicos de diferentes países o instituciones educativas, así como para el desarrollo de estrategias e instrumentos de evaluación (Bonanno, 2011).

En el ámbito español, el marco propuesto por el INTEF $(2013,2017)$, ha sido tomado como fundamento para diversas implementaciones autonómicas y locales, por ejemplo por la Consejería de Educación y Cultura del Gobierno de Extremadura (2015) al establecer su Portafolio de Competencia Digital Docente como sistema para la formación y el reconocimiento profesional de sus docentes. Asimismo, el modelo propuesto por la Generalitat de Catalunya (2016), ha servido también para el desarrollo derivado de rúbricas y estrategias de formación y evaluación (Lazaro, Gisbert, Palau, y Esteve, 2016).

Otros modelos, como el DigCompEdu (European Commission, 2017) o la reciente versión de los estándares de ISTE (2017), son todavía muy recientes para poder analizar su desarrollo derivado.

Entre los modelos de CDD citados los hay más centrados en las habilidades digitales de tipo instrumental y su aplicación en el aula y los hay que mencionan también otras funciones docentes, como la creación de recursos y entornos de aprendizaje, la colaboración y la participación, la organización y gestión de procesos o el desarrollo profesional docente (Esteve, 2015).

\subsection{Análisis descriptivo}

En la siguiente tabla se han situado los componentes esenciales (dimensiones, aspectos, funciones) de los siete marcos citados. Cada componente aparece de forma literal, tal cual se enuncia en el documento original. Además, se ha clasificado cada uno de dichos componentes en función de las categorías genéricas de la última columna, de acuerdo con el aspecto de la profesión docente a la que alude, a fin de destacar sus coincidencias y disparidades. 
Tabla 1. Componentes de los modelos institucionales de competencia digital.

\begin{tabular}{|c|c|c|c|c|c|c|c|}
\hline ISTE (2008)* & $\begin{array}{l}\text { UNESCO } \\
\text { (2011) }\end{array}$ & $\begin{array}{l}\text { DigCompEdu } \\
\text { (2017) }\end{array}$ & $\begin{array}{l}\text { ENLACES } \\
\text { (2011) }\end{array}$ & $\begin{array}{l}\text { DIGILiT } \\
\text { (2013) }\end{array}$ & INTEF (2017) & $\begin{array}{l}\text { Generalitat } \\
\text { de Catalunya } \\
\text { (2016) }\end{array}$ & \\
\hline & & $\begin{array}{c}\text { Compromiso } \\
\text { profesional: } \\
\text { gestión de datos }\end{array}$ & & $\begin{array}{l}\text { Búsqueda, } \\
\text { evaluación y } \\
\text { organización de } \\
\text { información }\end{array}$ & Información & & 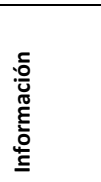 \\
\hline \multirow[t]{2}{*}{$\begin{array}{c}\text { Facilitar e } \\
\text { inspirar el } \\
\text { aprendizaje y la } \\
\text { creatividad de } \\
\text { sus alumnos } \\
\text { Diseñar y } \\
\text { desarrollar } \\
\text { experiencias de } \\
\text { aprendizaje y } \\
\text { evaluación } \\
\text { propias de la era } \\
\text { digital } \\
\end{array}$} & $\begin{array}{c}\text { Las políticas, el } \\
\text { plan de estudios } \\
\text { (currículum) y la } \\
\text { evaluación, la } \\
\text { pedagogía. }\end{array}$ & $\begin{array}{l}\text { Pedagogía } \\
\text { digital } \\
\text { Evaluación } \\
\text { digital }\end{array}$ & $\begin{array}{c}\text { Dimensión } \\
\text { pedagógica, } \\
\text { para la } \\
\text { integración de } \\
\text { las TIC en los } \\
\text { procesos de } \\
\text { enseñanza- } \\
\text { aprendizaje }\end{array}$ & $\begin{array}{c}\text { Evaluación y el } \\
\text { feedback } \\
\text { aprovechando } \\
\text { las posibilidades } \\
\text { de las TIC }\end{array}$ & & $\begin{array}{c}\text { Diseño, } \\
\text { planificación e } \\
\text { implementación } \\
\text { didáctica }\end{array}$ & 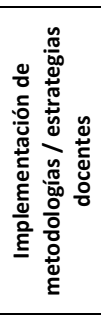 \\
\hline & Las TIC & & $\begin{array}{c}\text { Dimensión } \\
\text { técnica o } \\
\text { instrumental, } \\
\text { uso de sistemas } \\
\text { y herramientas } \\
\text { actuales }\end{array}$ & & $\begin{array}{c}\text { Resolución de } \\
\text { problemas }\end{array}$ & & 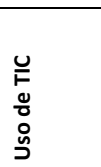 \\
\hline \multirow[t]{2}{*}{$\begin{array}{c}\text { Demostrar el } \\
\text { trabajo y el } \\
\text { aprendizaje } \\
\text { característicos } \\
\text { de la era digital }\end{array}$} & & $\begin{array}{l}\text { Recursos } \\
\text { Digitales }\end{array}$ & & $\begin{array}{c}\text { Creación, } \\
\text { reutilización y la } \\
\text { posibilidad de } \\
\text { compartir } \\
\text { recursos y } \\
\text { materiales en } \\
\text { línea }\end{array}$ & $\begin{array}{l}\text { creación de } \\
\text { contenidos }\end{array}$ & & 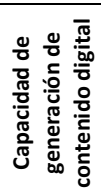 \\
\hline & $\begin{array}{l}\text { Organización y } \\
\text { administración }\end{array}$ & $\begin{array}{c}\text { Compromiso } \\
\text { profesional }\end{array}$ & $\begin{array}{l}\text { Gestión, } \\
\text { centrada en el } \\
\text { desarrollo de } \\
\text { procesos de } \\
\text { planificación } \\
\text { curricular y } \\
\text { gestión }\end{array}$ & & & $\begin{array}{l}\text { organización y } \\
\text { gestión de } \\
\text { espacios y } \\
\text { recursos } \\
\text { educativos }\end{array}$ & : \\
\hline $\begin{array}{c}\text { Promover y } \\
\text { demostrar la } \\
\text { ciudadanía } \\
\text { digital y la } \\
\text { responsabilidad }\end{array}$ & & $\begin{array}{c}\text { Compromiso } \\
\text { profesional } \\
\text { Empoderar a los } \\
\text { estudiantes }\end{array}$ & $\begin{array}{l}\text { Dimensión } \\
\text { social, ética y } \\
\text { legal; } y\end{array}$ & $\begin{array}{c}\text { Comunicación, } \\
\text { colaboración y } \\
\text { participación } \\
\text { La e-Seguridad e } \\
\text { identidad en } \\
\text { línea }\end{array}$ & $\begin{array}{c}\text { Comunicación } \\
\text { seguridad }\end{array}$ & $\begin{array}{c}\text { Comunicación y } \\
\text { colaboración } \\
\text { Ética y civismo } \\
\text { digital }\end{array}$ & 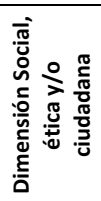 \\
\hline $\begin{array}{c}\text { Comprometerse } \\
\text { con el } \\
\text { crecimiento } \\
\text { profesional y el } \\
\text { liderazgo }\end{array}$ & $\begin{array}{c}\text { la formación } \\
\text { profesional de } \\
\text { docentes. }\end{array}$ & $\begin{array}{c}\text { Compromiso } \\
\text { profesional: } \\
\text { Desarrollo } \\
\text { profesional } \\
\text { continuo digital }\end{array}$ & $\begin{array}{l}\text { La dimensión de } \\
\text { desarrollo y } \\
\text { responsabilidad } \\
\text { profesional. }\end{array}$ & $\begin{array}{l}\text { La tecnología } \\
\text { como apoyo al } \\
\text { desarrollo } \\
\text { profesional del } \\
\text { docente. }\end{array}$ & & $\begin{array}{c}\text { Desarrollo } \\
\text { profesional. }\end{array}$ & 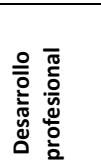 \\
\hline
\end{tabular}

(*) Se analiza la versión de 2008, ya que la de 2017, por su radical cambio de perspectiva, no encaja con los criterios de análisis.

Si atendemos a los aspectos generales de la CDD los marcos de la Tabla 1 (columna de la derecha), la mayoría de ellos (6 de los 7) incluyen la competencia del docente para usar las TIC eficientemente en la implementación de metodologías o estrategias docentes, para su propio desarrollo profesional, para la promoción de la colaboración, la participación, el "uso legal" de la red y la e-seguridad. Al menos la mitad de los modelos incluye una dimensión instrumental, relativa al uso de las TIC y la resolución autónoma de problemas técnicos. Otros incluyen el uso de las TIC para la gestión escolar y educativa y para la generación de contenidos. Tres modelos contienen, además, una dimensión referida a la búsqueda y gestión de la información online. 
Estas siete dimensiones parecen refrendar la tesis inicial de este estudio: casi todos los marcos se centran en el uso instrumental de la tecnología, en una acción docente profesional casi exclusivamente restringida a la enseñanza en la clase convencional y los aspectos éticos y sociales de la profesión docente se reducen a enseñar el uso legal de la tecnología o a la seguridad. Es más, a la luz del análisis anterior, pareciera que el común denominador de los marcos de la CDD se basa más en un docente que solo ejerce su profesión "en el aula" y que "domina la tecnología" entendida como mera herramienta sin más complicaciones. Es más, el modelo de competencia que subyace a estos marcos se aproxima mucho al concepto más restrictivo $\mathrm{y}$, a nuestro juicio, socialmente menos interesante, de competencia profesional.

\section{Discusión: Acción docente, competencia y tecnología}

Desde nuestra perspectiva, cualquier propuesta de CDD debería basarse en una serie de decisiones explícitas sobre temas complejos: qué se entiende por "competencia", qué hace un/a docente en el ejercicio de su profesión (y no solo en los procesos de enseñanza y aprendizaje en el aula) y qué visión de la tecnología se promueve. Sin embargo, esas decisiones -seguramente consecuencia de los propios tiempos que vivimos (Castañeda, 2016)- no suelen ser explicitadas, ni sus presupuestos suficientemente debatidos ni en los documentos publicados, ni en los procesos de desarrollo, de casi ninguno de los modelos analizados. Los marcos teóricos de la CDD, sea hayan debatido explícitamente o no, asumen implícitamente una serie de presupuestos que condicionan no solo su contenido (las dimensiones consideradas relevantes), sino también su estructura; e incluso las finalidades para las cuales han sido diseñados y los diferentes intereses a los que sirven no son ajenos a estas decisiones.

Por ello, porque entendemos que no se abordan suficiente o adecuadamente esos temas -al menos en los modelos analizados-, creemos que es necesario empezar por el principio y formular una nueva propuesta a debate de la CDD que responda, al menos, a tres preguntas: (a) ¿qué modelo educativo o de acción docente propone? (b) ¿qué tipo de competencia defiende? y (c) ¿qué visión de la tecnología asume?

\section{1.¿Cómo se concibe la acción docente?}

Para hacer un planteamiento sobre cuál debe ser la competencia digital de un docente o cómo debería concretarse la competencia docente en una sociedad crecientemente tecnológica, creemos prioritario precisar cuáles son los aspectos que componen la identidad docente.

Williamson (2013, p. 1) nos recuerda que no existe un concepto único de lo que es "un buen profesor", sino un amplio rango de ideas diferentes, en parte conflictivas, en parte "compatibles", producidas "por diferentes grupos en momentos diferentes". Discursos socialmente construidos que coexisten y que llegan a los docentes ens una cacofonía de propuestas e identidades. Sin embargo, entendemos que esas "cualidades profesionales fundamentales" que, en palabras de Pérez Gómez (2010, p. 49) deben servir al docente como "sistemas de comprensión y actuación profesional", deben definirse. Sin ellas es posible que la definición del docente competente en la era digital ignore algunos de los rasgos fundamentales de su acción docente.

Además de los marcos europeos de competencias docentes, analizados recientemente por Sanchez-Tarazaga (2016), existe abundante literatura científica dedicado

¿Por qué es necesario repensar la competencia docente para el mundo digital? Linda Castañeda, Francesc Esteve y Jordi Adell.

Página 7 de 20 
expresamente a la definición de la profesión docente y de sus competencias básicas (Caena, 2011, 2013). Por citar solo algunos, nos gustaría aludir a los modelos resultantes de estudios como el de Reynolds, Tannenbaum y Rosenfeld (1992) o a los trabajos de Shulman (1986). Este último puso en el centro de la discusión la necesaria armonización de los saberes pedagógico y de contenido (Content Knowledge y Pedagogical Knowledge) en el saber de profesor, dando lugar a los trabajos derivados, como los de Mishra y Koehler (2006), que amplían ese modelo con el conocimiento sobre tecnología (Technological Knowledge), formando el modelo TPACK (Technological, Pedagogical y Content Knowledge), uno de los modelos de referencia en los últimos años de la labor docente y la formación del profesorado.

A lo largo del tiempo han existido diversos paradigmas o discursos dominantes sobre el "buen docente". En The Good Teacher, Moore (2004) definió cuatro discursos: (a) el profesor carismático de los 80; (b) el artesano competente, dominante desde los 90 especialmente en la documentación político-educativa europea-; (c) el práctico reflexivo de la formación universitaria del profesorado, también desde los 90, y (d) el pragmático de los 2000, un docente "todoterreno" que se adapta a cualquier circunstancia. Además es necesario mencionar otros trabajos que, si bien no proponen un modelo como tal, sí que inciden en los rasgos característicos que sería necesario tener en cuenta, como los de Darling-Hammond (2006), Mishra y Koehler (2006) ya citado, Pérez Gómez (2010), Reynolds et al. (1992), Shulman (1986) o Tardif (2004) y que no es posible tratar aquí en detalle.

Ahora bien, la gran mayoría de estos planteamientos basan la acción docente en una competencia que incluye los aspectos didácticos y psicopedagógicos, el conocimiento sobre el contenido que se enseña, la capacidad de generación de contextos de aprendizaje y la capacidad para el desarrollo profesional docente. Sin embargo, existen otros enfoques que reclaman para el docente el papel social comprometido y crítico que proponía Perrenoud en el 2004. Por ejemplo, la articulación de las dimensiones estética y moral del saber del profesor (Rios, 2008), el compromiso social que reclama Martínez-Bonafé (2001) o las propuestas sobre las relaciones profesor-familiacomunidad desarrollados recientemente por Zeichner, Bowman, Guillen y Napolitan (2016) con base en las ideas de Cochran-Smith (2005).

Para llegar a ser un buen docente es necesario no solo dominar los contenidos, poseer las habilidades pedagógicas apropiadas y conocer los procedimientos habituales en el aula, sin duda aspectos sumamente importantes. También es "una cuestión de entender críticamente la enseñanza como una profesión que está siendo constantemente moldeada y remodelada de acuerdo con contextos históricos cambiantes, demandas políticas, representaciones de los medios de comunicación, intereses empresariales y evidencias de la investigación" (Williamson, 2013, p. 2). Una comprensión crítica del buen docente precisa tratar el concepto como "un producto social que se construye a través del discurso -formas legítimas de pensar, hablar y actuar como un docente- y a través de vocabularios, conceptos y teorías históricamente producidos"(Williamson, 2013, p. 8).

Desde nuestra perspectiva, el perfil del docente deseable se aproxima al modelo propuesto por Escudero (2005, pág. 12) cuando esquematiza las "creencias, conocimientos, capacidades y actitudes básicas de la identidad docente" y las concreta en un docente: (a) experto en contenidos, (b) experto en metodologías, (c) capaz de

¿Por qué es necesario repensar la competencia docente para el mundo digital? Linda Castañeda, Francesc Esteve y Jordi Adell.

Página 8 de 20 
aprender de su propia práctica, (d) que forma parte de su comunidad que aprende, (e) que establece una relación positiva con la familia y el entorno de los estudiantes, y (f) sensible a los imperativos sociales y éticos de su práctica. Seis partes de la competencia docente, íntimamente relacionadas entre sí, pero claramente diferenciadas, que incluyen conocimientos, habilidades y actitudes muy diferentes sin las cuales la acción docente pierde parte importante de su naturaleza.

Visto así, si volvemos al análisis de los aspectos que se tienen en cuenta en los modelos de CDD, podemos ver que casi todos ellos proponen el uso de la tecnología en los aspectos que podríamos llamar "clásicos" del perfil docente, pero ignoran prácticamente los aspectos referidos anteriormente y concretados en los dos últimos puntos del modelo propuesto por Escudero. Es decir, la mayoría de modelos abordan el conocimiento sobre el contenido (información y generación de contenido digital), conocimiento pedagógico (implementación de metodologías / estrategias docentes), conocimiento de gestión (gestión y uso de TIC) y desarrollo profesional, y, aunque nominalmente casi todos incluyen la "dimensión social, ética y/o ciudadana", esta se concreta en conocimientos sobre cómo usar redes sociales online, mecanismos para protegerse en la red y sobre el marco legal de uso de los materiales en red, lo cual dista mucho de los imperativos sociales y éticos de la profesión de la que hablábamos más arriba.

\section{2. ¿A qué modelo de competencia se sirve?}

El concepto de competencia que subyace a un modelo de competencia (digital o de cualquier otro tipo) define en gran manera la forma en que se concibe no sólo la competencia misma, sino el sistema en el que se inserta esa competencia y el tipo de cosas para las que se usará.

No obstante, dada la inmensa literatura referida a la conceptualización de las competencias, creemos que no resulta tan esclarecedor analizar todos los conceptos de competencia que subyacen a unos u otros modelos, sino analizar algunas dimensiones clave. Mulder, Weigel, y Collings (2008) distinguen una serie de dimensiones que incorporan todas las conceptualizaciones de competencia que se han utilizado en los últimos tiempos (Tabla 2).

Tabla 2. Dimensiones del concepto de competencia. Elaboración propia basada en (Mulder et al., 2008)

\begin{tabular}{|c|c|}
\hline $\begin{array}{l}\text { Entendido como una habilidad } \\
\text { periférica... }\end{array}$ & $\begin{array}{r}\text {...Entendido como una habilidad } \\
\text { principal }\end{array}$ \\
\hline Disgregado contextualmente... & ...Situado \\
\hline Orientado hacia roles... & ...Orientado a funciones \\
\hline Representado como conocimiento... & ..Representado como habilidades \\
\hline Focalizado en el comportamiento... & ...Focalizado en la capacidad \\
\hline La persona encarna la competencia... & $\begin{array}{r}\text {...El sistema encarna la } \\
\text { competencia }\end{array}$ \\
\hline Ámbito específico... & ...Ámbito general \\
\hline Entrenable... & ...Inalterable \\
\hline Orientado al desempeño... & $\begin{array}{r}\text {...Orientado al desarrollo de la } \\
\text { competencia }\end{array}$ \\
\hline
\end{tabular}

¿Por qué es necesario repensar la competencia docente para el mundo digital? Linda Castañeda, Francesc Esteve y Jordi Adell. 
Como es evidente, las dimensiones son independientes entre sí y no se presentan como opciones dicotómicas, sino que cada una de las conceptualizaciones de competencia pueden ubicarse en un punto del continuo de la dimensión. No sería demasiado complicado caracterizar el concepto de competencia que subyace a los modelos que hemos analizado en el apartado anterior como modelos técnicos de competencia (Beckett, 2008). Este análisis haría más evidente si cabe que, en términos de competencia docente, a mediados del siglo XX servíamos a los resultados, a principios del XXI servimos a la tecnología (Korthagen, 2004, p. 3), y es posible que llegados a este punto, y con todos los desarrollos en torno ala utilización de datos, en el futuro sirvamos a los resultados manejados por las tecnologías.

Pero entonces, ¿los marcos presentados en el apartado anterior definen la CDD de forma integral y holística? Entendemos que una competencia es mucho más que meros conocimientos y habilidades. Siguiendo a Perrenoud (2004), concebimos la competencia como un poder de acción que presupone la consecución de unos conocimientos y habilidades, pero que va más allá de ellos. La competencia implica también la facultad y el juicio para hacer uso de conocimientos y habilidades, movilizarlos, combinarlos y transferirlos para actuar de manera consciente y eficaz, con vistas a una finalidad éticamente deseable (Escudero, 2009).

Caracterizándola con las dimensiones de la Tabla 2, diríamos que consideramos deseable que se articule un modelo de competencia docente para el mundo digital:

- Que entienda la competencia como habilidad principal, en tanto que define la identidad del profesor desde una visión holística de su ejercicio profesional (Korthagen, 2004).

- Situado, es decir, que no pueda desentenderse del contexto en el que se desarrolla y no entienda ese contexto como un mero marco, sino como parte integral de su definición.

- Orientado hacia roles de desempeño, función y relación del profesorado.

- Representado como conocimientos, pero entendidos de forma amplia (conceptos, habilidades y actitudes) y no como "micro habilidades instrumentales".

- Que dibuje un sistema de desempeño en el que el docente tiene un lugar, pero en el que no es el único actor competente. Es decir, no es el único agente, ni el único responsable de su competencia, sino un actante clave en las relaciones que configuran esa competencia, una visión socio-material de la competencia (Decuypere y Simons, 2016).

- Que debe definirse para cada ámbito específico de la educación y no quedarse en posiciones excesivamente generalistas. Estamos convencidos de que, al menos en lo que se refiere a educación, cuando se enuncian modelos generalistas partimos de premisas iniciales y fundamentales (p.e. ¿cuál es el propósito de la educación básica o cuál es el propósito de la educación superior?) que, al variar, hacen los modelos tan generales que no ayudan en la definición de ninguna de las realidades pretendidas, incomparables en su composición o simplemente inadecuadas para todos los ámbitos en los que podrían implementarse.

- Educable, siempre con posibilidades de ser desarrollada, aunque no como un conjunto de habilidades discretas que pueden desligarse unas de otras y entrenarse de forma individual y descontextualizada... no intentando que la

¿Por qué es necesario repensar la competencia docente para el mundo digital? Linda Castañeda, Francesc Esteve y Jordi Adell.

Página 10 de 20 
mente del profesor "refleje" la competencia, tanto como que se ilumine con ella (Beckett, 2008).

- Orientado al desarrollo de la competencia y no solo al desempeño, porque no concebimos la competencia del profesor como un estado final, sino como una permanente mejora, análoga al concepto de "escuela que aprende" (Bolivar, 2001).

\section{3. ¿Qué visión de la tecnología subyace a estos modelos?}

Uno de los presupuestos básicos de todo modelo de CDD es su visión del papel de la tecnología como mediador de la acción humana y, concretamente de la acción educativa. Un instrumentalismo optimista, que podríamos calificar de entusiástico, late en la base de la mayor parte del discurso tecnológico-educativo sobre las "posibilidades" educativas de las TIC. La manera de entender la naturaleza de la tecnología subyace a cómo los docentes se acercan a la tecnología educativa especialmente con la reciente-, cómo diseñan actividades didácticas utilizando las TIC, cómo se planean, implementan y evalúan políticas educativas respecto a las TIC (proyectos "uno-a-uno", por ejemplo), cómo se "recibe" y valora la innovación didáctica con TIC o cómo se define y juzga la propia "competencia". Desgraciadamente, la tecnología educativa carece prácticamente de teorización (Bulfin, Henderson y Johnson, 2013).

El debate sobre el impacto de la tecnología en la sociedad es tan antiguo como el propio pensamiento: no hace falta recordar aquí la opinión de Platón en el Fedro sobre la escritura. Las teorías modernas de la tecnología se pueden representar en un continuo entre dos extremos: el determinismo tecnológico y el instrumentalismo tecnológico (Adell, en prensa).

Los autores que se posicionan cerca del extremo determinista ven la tecnología como la causa principal del cambio social y la sitúan más allá del control humano. Para los deterministas, el desarrollo tecnológico es un proceso discontinuo que procede mediante saltos o revoluciones que lo cambian todo. Existen dos variedades puras de determinismo tecnológico: la utópica (la tecnología es una fuerza de progreso inevitable y autónoma que conducirá a la prosperidad y será la salvación de la humanidad, incluso de los problemas que ha creado la propia tecnología) y la distópica (la tecnología es una fuerza inevitable y autónoma, moralmente corrupta, que conducirá indefectiblemente a la destrucción de la humanidad) (Surry y Farquhar, 1997). Los instrumentalistas tecnológicos, por su parte, explican la tecnología asimilándola al concepto de herramienta, siempre bajo control humano, que puede ser utilizada tanto para el bien como para el mal en función de las intenciones de quien la usa. Desde el instrumentalismo, el cambio es producto de la voluntad humana y el desarrollo tecnológico es gradual y progresivo.

Feenberg (1999) propuso una dimensión más en la que difieren las visiones clásicas de la tecnología: los valores. En un extremo, la convicción de que la tecnología es neutral, un medio, es decir, que en sí misma no "acarrea" ni impone los valores de las decisiones de diseño y puede ser utilizada de cualquier forma que decida el usuario. Y en el otro, la tecnología está cargada de valores y, como medio, no es independiente de los fines para los que ha sido creada. El sustantivismo de Ellul (1980) sería un ejemplo de determinismo distópico cargado de valores. La teoría crítica de la tecnología (Feenberg,

¿Por qué es necesario repensar la competencia docente para el mundo digital? Linda Castañeda, Francesc Esteve y Jordi Adell.

Página 11 de 20 
1999), en cambio, propondría una visión en la que la tecnología está cargada de valores, pero también es, hasta cierto punto, humanamente controlable.

Sin entrar en más detalles, la mayor parte de la investigación actual sobre tecnología educativa y las descripciones de la CDD asumen una perspectiva instrumentalista a nivel micro (la tecnología es neutral, los seres humanos la controlamos y, por tanto, la tecnología en educación "solo es una herramienta" al servicio de los docentes, que pueden emplear como quieran o al servicio de los fines de la educación que decidan) y, al mismo tiempo, promueve una visión determinista a nivel macro, que ve inevitable y ya trazado el camino, etiquetado como progreso y quintaesenciado en conceptos como el de sociedad de la información (o del conocimiento) o las "competencias del siglo XXI") y la necesidad de que el sistema educativo y los docentes se "adapten" al ritmo del cambio de los desarrollos tecnológicos (Webster, 2017). El discurso hegemónico de la tecnología educativa pretende extender la convicción de la inevitabilidad de un futuro inherente a la lógica interna de la propia tecnología, es decir, sin ideología, que hace imprescindible capacitar a los docentes para utilizar ciertas tecnologías y no otras, de ciertas formas y para ciertos fines y no otros.

Desde otras maneras de "pensar" la tecnología (Feenberg (1999), Latour (2008) o Winner (1980 y 2001), por citar algunos ejemplos) se ha intentado superar estos dualismos irreconciliables. En un contexto educativo, Oliver (2016) recoge una serie de debates contemporáneos que podrían enriquecer la "empobrecida comprensión de la tecnología... que es muy visible en la política educativa contemporánea, así como en la investigación" (pág. 40). En concreto Oliver anima a pensar en la tecnología como causa, la tecnología como intervención social, la tecnología como reificación de prácticas sociales, la tecnología como instanciación de la teoría, la tecnología como sistema dentro de sistemas y, finalmente, en la tecnología como efecto red.

Todos estos debates rehuidos condicionan tanto la teorización como la práctica educativa, que siempre es "con tecnología" aunque ya no la veamos. No plantearlos implica rendir la tecnología educativa a la visión (y la agenda) de un interesado optimismo tecnológico. Frente a la riqueza y complejidad de estas perspectivas, las descripciones de la CDD dominantes se basan, no extrañamente, en una visión de la tecnología como un instrumento neutro para los docentes, pero, al mismo tiempo, en una perspectiva determinista, y a veces resignada, sobre la inevitabilidad del cambio.

\section{Un marco de competencia docente integral en el mundo digital para la educación básica: una primera aproximación}

Tomando como punto de partida el análisis de los apartados previos, y fieles a una vocación didáctica que pretende ir más allá de la mera enunciación de las carencias de modelos anteriores, proponemos tentativamente y a debate un modelo que intenta solventar los problemas detectados y encarnar las características que enunciábamos en los apartados anteriores.

Se trata de un modelo específicamente pensado para la educación básica, que presentamos a continuación en su forma gráfica y que caracterizaremos sucintamente en sus componentes principales. Un modelo que se aborda de forma más detallada en trabajos anteriores ((Esteve, Castañeda, y Adell, 2018)

¿Por qué es necesario repensar la competencia docente para el mundo digital? Linda Castañeda, Francesc Esteve y Jordi Adell.

Página 12 de 20 


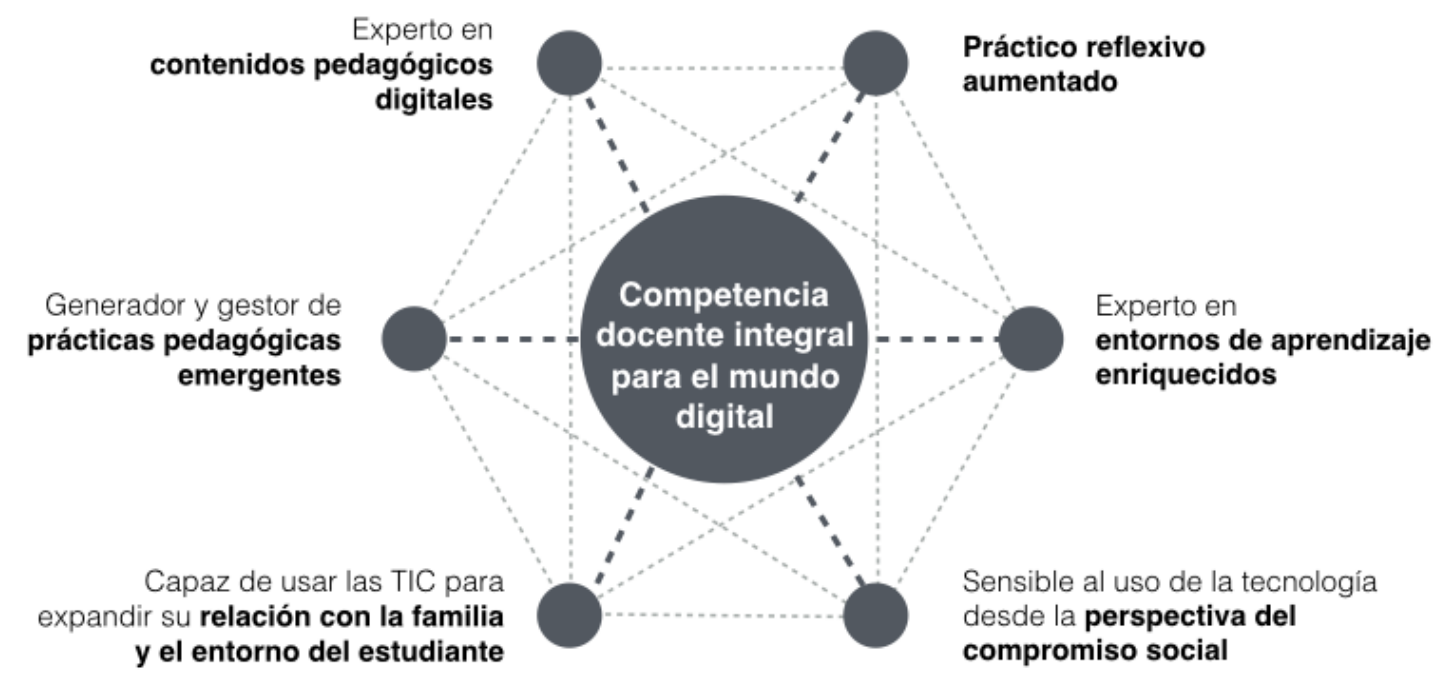

Figura 1. Modelo de Competencia Docente Integral en el mundo digital. (Esteve, Castañeda y Adell, 2018)

Así, entendemos la competencia docente integral en el mundo digital para la educación básica, como una competencia que se encarna en un profesor:

a) Generador y gestor de prácticas pedagógicas emergentes

Capaz no solo de usar las TIC para enriquecer sus estrategias didácticas habituales, sino que sería capaz de proponer y realizar sus propios modelos metodológicos, sus propias prácticas emergentes con tecnología.

b) Experto en contenidos pedagógicos digitales

Capaz de usar la tecnología para introducir uno u otro aspecto del aprendizaje de un contenido y, sobre todo, de articular el uso de la tecnología en la organización y desarrollo de estrategias didácticas específicas que permitan el desarrollo de competencias concretas en los estudiantes.

c) Práctico reflexivo aumentado

Capaz de "permitir" que las tecnologías y los procesos típicos de la investigación en el mundo digital permeen en cada una de las fases de esa práctica reflexiva sistematizada.

d) Experto en entornos enriquecidos de aprendizaje personal y organizativos Capaz de aprender eficientemente, de forma autónoma, en red (Entornos Personales de Aprendizaje) y junto a sus colegas (Entornos Organizativos de Aprendizaje) aprovechando los medios y oportunidades que ofrece el contexto tecnológico actual.

e) Sensible al uso de la tecnología desde la perspectiva del compromiso social Capaz de entender el papel de la tecnología como herramienta de compromiso social. Consciente del potencial de esas tecnologías para el cambio social.

f) Capaz de usar la tecnología para expandir su relación con la familia y el entorno del estudiante

Un agente comprometido con el estudiante que utiliza las TIC para ayuda a coordinar los esfuerzos educativos de los entornos determinantes del educando (familia, amigos, centro, barrio).

¿Por qué es necesario repensar la competencia docente para el mundo digital? Linda Castañeda, Francesc Esteve y Jordi Adell. 


\section{Conclusiones}

Los marcos institucionales de la CDD que hemos analizado son modelos performativos de evaluación, control y formación en destrezas técnicas básicas, casi siempre en ese orden y con un especial énfasis en la naturaleza individual del profesor como profesional.

Bien es cierto que puede parecer que esos desarrollos son ineludibles si queremos que un modelo de competencia sea de utilidad ("si no se evalúa, se pierde", "definir el modelo de docente que queremos es crucial para que la educación...", "la base es la formación del profesorado", "los modelos sirven como guía de la formación", etc.). Sin embargo, esos desarrollos de "utilidad" distan mucho de ser uniformes o equivalentes en todos los modelos (no todos los modelos/marcos de competencias deben materializarse en una rúbrica por niveles $\mathrm{o}$ en habilidades discretas $\mathrm{y}$ descontextualizadas), sino que son consecuencia del concepto mismo de competencia que subyace a cada uno de esos modelos, sea explícito o no (Hager, 1995).

A lo largo de este trabajo hemos intentado justificar la necesidad de un modelo que deje de hablar de la "competencia digital del docente" como si el docente fuese "divisible" en trozos digitales y otros que no lo son y apostar por un modelo docente renovado, que responda adecuadamente al contexto en el que se enmarca y que se materialice en forma de competencia. Una competencia docente para el mundo digital entendida como holística, situada, orientada hacia roles de desempeño, función y relación, sistémica, entrenable y en constante desarrollo. Un modelo que tiene en cuenta que la tecnología es un instrumento al servicio de fines educativos, pero también un es un tipo de conocimiento y de relación con el mundo, una actividad humana y una fuente de valores (de Vries, 2016). El docente competente entiende que ni él ni el estudiante son los "dueños" omnipotentes de esa tecnología, sino usuarios situados en un marco sistémico de condicionamientos, pero usuarios conscientes de su vulnerabilidad y de la necesidad de empoderamiento crítico y de la posibilidad de desarrollar su agencia, entendida como capacidad técnica guiada por el buen juicio, en mundo crecientemente mediado por la tecnología.

Entendemos que a esas características responde el modelo que presentamos, pero lo cierto es que es solo un paso que requerirá otros más y debates más profundos en la práctica de la competencia de los actores educativos para responder adecuadamente al mundo digital.

Presentación del artículo: 6 de noviembre de 2017

Fecha de aprobación: 9 de enero de 2018

Fecha de publicación: 31 de enero de 2018

Castañeda, L., Esteve, F. y Adell, J. (2018). ¿Por qué es necesario repensar la competencia docente para el mundo digital? RED. Revista de Educación a Distancia, 56. Consultado el (dd/mm/aaaa) en http://www.um.es/ead/red/56/castaneda_et_al.pdf

¿Por qué es necesario repensar la competencia docente para el mundo digital? Linda Castañeda, Francesc Esteve y Jordi Adell. 


\section{Financiación}

Esta investigación no ha recibido ninguna subvención específica de los organismos de financiación en los sectores públicos, comerciales o sin fines de lucro.

\section{Referencias bibliográficas}

Adell, J. (en prensa). Más allá del instrumentalismo en tecnología educativa. En Gimeno-Sacristán, J. (ed.). El vaciado de las palabras en educación. Madrid: Morata.

Álvarez, J. F. y Gisbert, M. (2015). Grado de alfabetización informacional del profesorado de Secundaria en España: Creencias y autopercepciones, Information Literacy Grade of Secondary School Teachers in Spain - Beliefs and Self-Perceptions. Comunicar: Revista Científica de Comunicación y Educación, Comunicar: Media Education Research Journal, 23(45), 187-194. https://doi.org/10.3916/C45-2015-20

Banister, S. y Reinhart, R. V. (2012). Assessing NETS - T Performance in Teacher Candidates. Journal of Digital Learning in Teacher Education, 29(2), 59-65. https://doi.org/10.1080/21532974.2012.10784705

Beckett, D. (2008). Holistic competence: Putting judgements first. Asia Pacific Education Review, 9(1), 21-30. https://doi.org/10.1007/BF03025822

Bolivar, A. (2001). Los centros educativos como organizaciones que aprenden: Una mirada crítica. Contexto Educativo-Revista Digital de Educación y Nuevas Tecnologias, 3(18).

Bonanno, P. (2011). Developing an instrument to assess teachers' readiness for technology-enhanced learning. En Interactive Collaborative Learning (ICL), 2011 14th International Conference on (pp. 438-443). IEEE. Recuperado a partir de http://ieeexplore.ieee.org/abstract/document/6059622/

Bulfin, S., Henderson, M. y Johnson, N. (2013). Examining the use of theory within educational technology and media research. Learning, Media and Technology, 38(3), 337-344. https://doi.org/10.1080/17439884.2013.790315

Caena, F. (2011). Literature review Quality in Teachers' continuing professional development. Education and training, 2020. Recuperado a partir de https://pdfs.semanticscholar.org/11c9/e90f3fb8a97e463882d5ab0846b2373279 a2.pdf

Caena, F. (2013). Supporting teacher competence development: For better learning outcomes. European Commission. Education and Training. Recuperado a partir de

http://ec.europa.eu/dgs/education_culture/repository/education/policy/school/d oc/teachercomp_en.pdf

Carrera, X., \& Coiduras Rodríguez, J. L. (2012). Identificación de la competencia digital del profesor universitario: un estudio exploratorio en el ámbito de las Ciencias Sociales. Red-U: Revista de docencia universitaria, 10 (2), 273-298.

Castañeda, L. (2016) Suelo y sol: más pensamiento y contexto para la innovación educativa. Boletín de la Institución Libre de Enseñanza, 104. 37-50. Recuperado a partir de http://hdl.handle.net/10201/54439

¿Por qué es necesario repensar la competencia docente para el mundo digital? Linda Castañeda, Francesc Esteve y Jordi Adell. 
Cochran-Smith, M. (2005). Teacher Development and Educational Reform. En M. Fullan (Ed.), Fundamental Change (pp. 246-281). Nueva York: Springer. https://doi.org/10.1007/1-4020-4454-2_14

Consejería de Educación y Cultura. Gobierno de Extremadura. RESOLUCIÓN de 2 de junio de 2015, de la Secretaría General de Educación, por la que se publica el Porfolio de Competencia Digital Docente de Extremadura., Pub. L. No. 2015061253, DOE NÚMERO 112 Viernes, 12 de junio de 201522961 (2015). Recuperado a partir de https://recursos.educarex.es/pdf/porfolio/porfoliopublicadoendoe.pdf

Departament d'Ensenyament. (2016). Resolució ENS/1356/2016, de 23 de maig, per la qual es dóna publicitat a la definició de Competència Digital. Diari Oficial de la Generalitat de Catalunya.

Darling-Hammond, L. (2006). Constructing 21st-Century Teacher Education. Journal of Teacher Education, 300-314. https://doi.org/10.1177/0022487105285962

de Vries, M. J. (2016). Teaching about technology : an introduction to the philosophy of technology for non-philosophers. Dordtecht, The Netherlands: Springer.

Decuypere, M. y Simons, M. (2016). Sobre el potencial crítico de los enfoques sociomateriales en educación. Teoría de la Educación. Revista Interuniversitaria, 28(1), 25. https://doi.org/10.14201/teoredu20162812544

Durán, M., Gutiérrez, I. y Prendes, M. P. (2016). Análisis conceptual de modelos de competencia digital del profesorado universitario. RELATEC: Revista Latinoamericana de Tecnología Educativa, 15(1), 97-114. https://doi.org/10.17398/1695-288X.15.1.97

Ellul, J. (1980). The Technological System. New York: Seabury Pr.

Enlaces. (2011). Competencias y Estándares TIC para la Profesión Docente. Centro de Educación y Tecnología (Enlaces). Ministerio de Educación, Gobierno de Chile. Recuperado a partir de http://www.enlaces.cl/download/competenciasdocentes/?wpdmdl=11947

Enlaces. (2013). Desarrollo de habilidades digitales para el siglo XXI en Chile: ¿Qué dice el SIMCE TIC? Santiago, Chile: Ministerio de Educación. Recuperado a partir de www.enlaces.cl

Escudero, J. M. (2005). El profesor y su formación: Competencias y formación docente al servicio de un modelo de escuela en nuestro tiempo. Programa y desarrollo temático de Formación y Actualización de la Función Pedagógica, 84-108.

Escudero, J. M. (2009). Las competencias profesionales y la formación universitaria: posibilidades y riesgos. Pedagogía Social. Revista Interuniversitaria, O(16), 65-82. https://doi.org/10.7179/PSRI_2009.16.05

Esteve, F. (2015, marzo 2). La competencia digital docente: análisis de la autopercepción y evaluación del desempeño de los estudiantes universitarios de educación por medio de un entorno 3D (Ph.D. Thesis). Universitat Rovira i Virgili. Recuperado a partir de http://www.tdx.cat/handle/10803/291441

¿Por qué es necesario repensar la competencia docente para el mundo digital? Linda Castañeda, Francesc Esteve y Jordi Adell. 
Esteve, F., Castañeda, L. y Adell, J. (2018). Un modelo holístico de competencia docente para el mundo digital. Revista Interuniversitaria de Formación del Profesorado, 32(1).

Esteve, F., Cela, J. M. y Gisbert, M. (2016). ETeach3D: Designing a 3D Virtual Environment for Evaluating the Digital Competence of Preservice Teachers. Journal of Educational Computing Research, 54(6), 816-839. https://doi.org/10.1177/0735633116637191

Esteve, F. y Gisbert, M. (2013). Competencia digital en la educación superior: instrumentos de evaluación y nuevos entornos. Enl@ce: Revista Venezolana de Información, Tecnología y Conocimiento, 10(3), 29-43. Recuperado a partir de http://www.redalyc.org/articulo.oa?id=82329477003

European Commission. (2017, 12). Learning to swim in the Digital Ocean: new DigComp report develops proficiency levels in detail. Recuperado 11 de octubre de 2017, a partir de https://ec.europa.eu/jrc/en/science-update/newdigcomp-report-develops-proficiency-levels

Feenberg, A. (1999). Questioning Technology. London; New York: Routledge.

Ferrari, A. (2013). DIGCOMP: A framework for developing and understanding digital competence in Europe. Sevilla: Institute for Prospective Technological Studies (IPTS), European Commission. Recuperado a partir de http://publications.jrc.ec.europa.eu/repository/bitstream/JRC83167/lb-na26035-enn.pdf

Fraser, J., Atkins, L. y Hall, R. J. (2013). DigiLit leicester. Supporting teachers, promoting digital literacy, transforming learning. Leicester City Council. Recuperado a partir de http://fraser.typepad.com/files/digilit-leicester-report130624-final.pdf

Hager, P. (1995). Competency Standards a Help or a Hindrance? An Australian Perspective. The Vocational Aspect of Education, 47(2), 141-151. https://doi.org/10.1080/0305787950470203

Hall, R., Atkins, L. y Fraser, J. (2014). Defining a self-evaluation digital literacy framework for secondary educators: the DigiLit Leicester project. Research in Learning Technology, 22(1), 21440. https://doi.org/10.3402/rlt.v22.21440

INTEF. (2013). Marco común de competencia digital docente (Borrador). Ministerio de Educación, Cultura y Deporte. Recuperado a partir de http://educalab.es/documents/10180/12809/MarcoComunCompeDigiDoceV2.p df

INTEF. (2017). Marco Común de Competencia Digital Docente. Ministerio de Educación, Cultura y Deporte. Recuperado a partir de http://blog.educalab.es/intef/2016/12/22/marco-comun-de-competencia-digitaldocente-2017-intef

ISTE. (2008). National Educational Technology Standards for Teachers. Washington DC: International Society for Technology in Education.

ISTE. (2017). ISTE Standards for Educators. USA: International Society for Technology in Education.

¿Por qué es necesario repensar la competencia docente para el mundo digital? Linda Castañeda, Francesc Esteve y Jordi Adell. 
Korthagen, F. A. J. (2004). In search of the essence of a good teacher: towards a more holistic approach in teacher education. Teaching and Teacher Education, 20(1), 77-97. https://doi.org/10.1016/j.tate.2003.10.002

Krumsvik, R. (2009). Situated learning in the network society and the digitised school. European Journal of Teacher Education, 32(2), 167-185. https://doi.org/10.1080/02619760802457224

Krumsvik, R. J. (2012). Teacher educators' digital competence. Scandinavian Journal of Educational Research, 58(3), 269-280. https://doi.org/10.1080/00313831.2012.726273

Kumar, S. y Vigil, K. (2011). The Net Generation as Preservice Teachers. Journal of Digital Learning in Teacher Education, 27(4), 144-153. https://doi.org/10.1080/21532974.2011.10784671

Latour, B. (2008). Reensamblar lo social: una introducción a la teoría del actor-red. Buenos Aires: Manantial.

Lazaro, J. L., Gisbert, M., Palau, R. y Esteve, V. (2016). Una estratègia per a la formació i avaluació de la competència digital dels docents. Revista del Congrés Internacional de Docència Universitària i Innovació (CIDUI), O(3). Recuperado a partir de http://www.cidui.org/revistacidui/index.php/cidui/article/view/866

Martínez Bonafé, J. (2001). Arqueología del concepto «compromiso social» en el discurso pedagógico y de formación docente. Revista Electrónica de Investigación Educativa (REDIE), 3(1). Recuperado a partir de http://www.redalyc.org/articulo.oa?id=15503106

Mishra, P. y Koehler, M. J. (2006). Technological pedagogical content knowledge: A framework for teacher knowledge. Teachers college record, 108(6), 1017. Recuperado a partir de http://onlinelearningcurriculumcommittee.pbworks.com/f/mishra.pdf

Mishra, P., Koehler, M. J. y Henriksen, D. (2011). The Seven Trans-Disciplinary Habits of Mind: Extending the TPACK Framework towards 21st Century Learning. Educational Technology, 51(2), 22-28.

Moore, A. (2004). The good teacher: Dominant discourses in teaching and teacher education. London y New York: RoutledgeFalmer.

Morphew, V. N. (2012). A Constructivist Approach to the National Educational Technology Standards for Teachers. United States: International Society for Technology in Education (ISTE).

Mulder, M., Weigel, T. y Collings, K. (2008). El concepto de competencia en el desarrollo de la educación y formación profesional en algunos Estados miembros de la UE: un análisis crítico. Profesorado: Revista de curriculum y formación del profesorado, 12(3), 1-25. Recuperado a partir de http://www.redalyc.org/pdf/567/56712875007.pdf

Oliver, M. (2016). What is Technology. En N. Rushby y D. Surry (Eds.), Wiley Handbook of Learning Technology (Edición: 1). Chichester, UK; Malden, MA: Wiley Handbooks in Education.

¿Por qué es necesario repensar la competencia docente para el mundo digital? Linda Castañeda, Francesc Esteve y Jordi Adell. 
Pérez Gómez, A. I. (2010). Aprender a educar: nuevos desafíos para la formación de docentes. Revista Interuniversitaria de Formación del Profesorado, 68(24,2), 37-60. Recuperado a partir de http://www.redalyc.org/html/274/27419198003/

Perrenoud, P. (2004). Desarrollar la práctica reflexiva en el oficio de enseñar: profesionalización y razón pedagógica. Barcelona: Graó.

Pettersson, F. (2017). On the issues of digital competence in educational contexts - a review of literature. Education and Information Technologies, 1-17. https://doi.org/10.1007/s10639-017-9649-3

Prendes, M. P. y Gutiérrez, I. (2013). Competencias tecnológicas del profesorado en las Universidades españolas. Revista de Educación, 361, 196-222. Recuperado a partir de https://recyt.fecyt.es/index.php/Redu/article/view/11814

Reynolds, A., Tannenbaum, R. J. y Rosenfeld, M. (1992). Beginning Teacher Knowledge of General Principles of Teaching and Learning: A National Survey. ETS Research Report Series, 1992(2), 16. https://doi.org/10.1002/j.2333-8504.1992.tb01491.x

Rios, T. A. (2003). Comprender y Enseñar: Por una docencia de la mejor calidad (Vol. 185). Mexico: Graó.

Salkind, N. (2018). Exploring Research. Essex: Pearson.

Sanchez-Tarazaga, L. (2016). Los marcos de competencias docentes: contribución a su estudio desde la política educativa europea. Journal Of Supranational Policies Of Education, 44-67. https://doi.org/http://dx.doi.org/10.15366/jospoe2016.5

Shulman, L. S. (1986). Those who understand: Knowledge growth in teaching. Educational researcher, 15(2), 4-14. https://doi.org/10.3102/0013189X015002004

Recuperado a partir de http://www2.gsu.edu/ wwwitr/docs/diffusi /

Tardif, M. (2004). Los saberes del docente y su desarrollo profesional. (P. M. Bernárdez, Trad.) (Edición: 1). Madrid: Narcea Ediciones.

Touriñan, J. M. (2001). Tecnología digital y sistema educativo: el reto de la globalización. Revista de educación, (1), 217-230. Recuperado a partir de https://www.mecd.gob.es/revista-deeducacion/numeros-revista-educacion/numeros-anteriores/2001/re2001.html.

UNESCO. (2008). Competency Standards Modules. ICT Competency Standards for Teachers. París: author. Recuperado a partir de http://unesdoc.unesco.org/images/0015/001562/156207e.pdf

UNESCO. (2011). UNESCO ICT Competency Framework for Teachers. Paris: United Nations Educational, Scientific and Cultural Organization (UNESCO).

UNESCO. (2014). ICT in Education. Recuperado a partir de http://www.unesco.org/new/en/unesco/themes/icts/

Webster, M. D. (2017). Philosophy of Technology Assumptions in Educational Technology Leadership. Educational Technology y Society, 20(1), 25-36. Recuperado a partir de http://www.jstor.org/stable/jeductechsoci.20.1.25

¿Por qué es necesario repensar la competencia docente para el mundo digital? Linda Castañeda, Francesc Esteve y Jordi Adell. 
Williamson, B. (2013). Making up the good teacher. Recuperado a partir de http://www.academia.edu/4459318/Making_up_the_good_teacher

Winner, L. (1980). Do artifacts have politics? Daedalus, 121-136.

Winner, L. (2001). The whale and the reactor: a search for limits in an age of high technology (Nachdr.). Chicago: Univ. of Chicago Press.

Zeichner, K., Bowman, M., Guillen, L. y Napolitan, K. (2016). Engaging and Working in Solidarity With Local Communities in Preparing the Teachers of Their Children. Journal of Teacher Education, 67(4), 277-290. https://doi.org/10.1177/0022487116660623 